
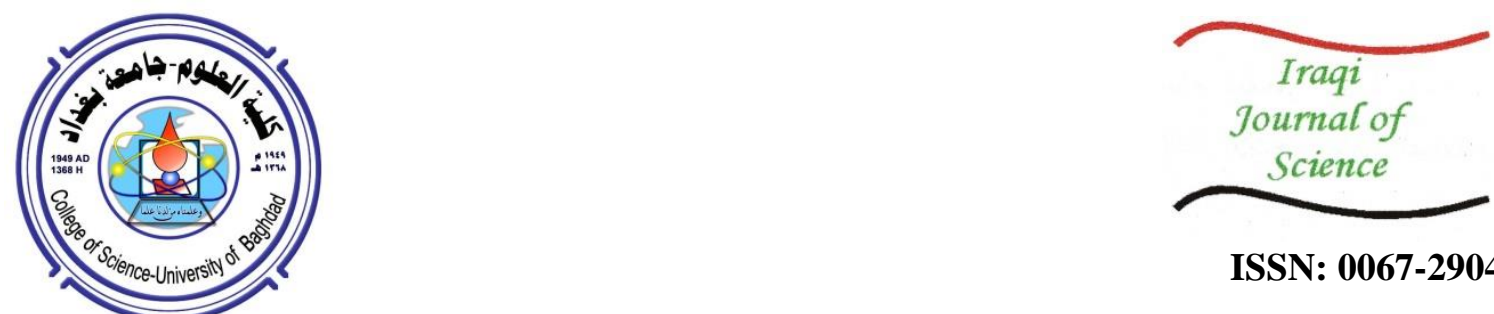

ISSN: 0067-2904

\title{
Splitting of $P G(1,27)$ by Sets, Orbits, and Arcs on the Conic
}

\author{
Emad Bakr Abdulkareem \\ Department of Mathematics, College of Science, Mustansrhiya University, Baghdad, Iraq
}

Received: $21 / 8 / 2019$

Accepted: 21/11/2020

\begin{abstract}
This research aims to give a splitting structure of the projective line over the finite field of order twenty-seven that can be found depending on the factors of the line order. Also, the line was partitioned by orbits using the companion matrix. Finally, we showed the number of projectively inequivalent $k$-arcs on the conic $v\left(X_{1}^{2}-X_{0} X_{2}\right)$ through the standard frame of the plane $P G(2,27)$.
\end{abstract}

Keywords: Conic, Finite field, Finite projective space.

$$
\begin{aligned}
& \text { تقسيم PG (1, 27 بواسطة المجموعات والمدارات, والأقواس على المخروط }
\end{aligned}
$$

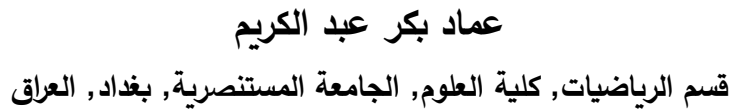

$$
\begin{aligned}
& \text { يهذف البحث إلى إعطاء بنية تقسيم للخط الإسقاطي على الحقل المنتهي ذات الرتبة سبعة وعشرين } \\
& \text { والتي يمكن العثور عليه اعتمادًا على عوامل رتبة الخط. أيضا، تم تقسيم الخط بواسطة المدارات باستخدام }
\end{aligned}
$$

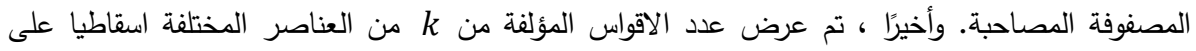

$$
\begin{aligned}
& \text { المخروط المار بالإطار القياسي للمستوي }
\end{aligned}
$$

\section{Introduction}

The partitions of the finite projective line into disjoint subsets which have certain characteristics depend on the order of the line, if it is prime number or not; that is, if it is factoring into non-trivial integer factorization. The factoring numbers of the line's order can also be used to split the projective line by fixed it as a power of the companion matrix (cyclic projectivity). Then, the line is partitioned by the orbits that come from the action of the companion matrix on the projective line. The partitionining of the line by orbits is regarded as a special approach, since the distinct orbits are projectively equivalent.

In [1], Hirschfeld discussed the projective line for arbitrary $q$ and then the certain types of splitting of projective line over $F_{q}, q=4,5,6,7,9,11,13$ were discussed. The projective line over $F_{19}$ was investigated and partitioned [2]. For $q=16,17$, the projective lines were only classified by Al-Seraji [3] and [4]. Al-Zangana [5] gave a classification and separated the projective line through the arcs on the conic when $q=23$. Later on, the line $P G(1,25)$ was classified and some partitions were given on it [6]. As related to the line $P G(1,27)$, it was only classified by Ibrahim [7], who gave the description of an $k$-set into the types of the $(k-1)$-subsets, $k=5, \ldots 14$. Among the researchers who focused only on the projective space over $F_{27}$, Coolsaet [8] o computed the complete arcs in the plane.

*Email: emad77_kaka@yahoo.com 
The aims of this paper are divided into two parts. The first discusses the most common ways to partition the line by taking advantage of the number twenty-seven, which achieved by using classical partitions and groups acting on the projective line. The second introduces the incomplete $\mathrm{k}$-arcs passing through the standard frame on the conic $C^{*}=v\left(X_{1}^{2}-X_{0} X_{2}\right)$.

\section{Basic Definitions and Previous Results}

Let $P G(n-1, q)$ denotes the $(n-1)$-dimensional projective space over the finite field of $q=p^{h}$ elements denoted by $F_{q}$. Let $F_{q}^{+}$denotes the field $F_{q}$ with an additional point $\infty$.

Definition 2.1 [1]: A $k$-arc, $K$ in the projective plane $P G(2, q)$, is a set of $k$ points where no three of them are collinear, but there are some two collinear. A $k$-set, $K$ in the projective line $\mathrm{PG}(1, \mathrm{q})$, is just a set of $k$ distinct points.

Definition 2.2 [1]: The cross-ratio of a set $S$ of four ordered points $P_{1}, P_{2}, P_{3}, P_{4} \in P G(1, q)$ with coordinates $t_{1}, t_{2}, t_{3}, t_{4}$ is

$$
C R(S)=\left\{P_{1}, P_{2} ; P_{3}, P_{4}\right\}=\left\{t_{1}, t_{2} ; t_{3}, t_{4}\right\}=\left(t_{1}-t_{3}\right)\left(t_{2}-t_{4}\right) /\left(t_{1}-t_{4}\right)\left(t_{2}-t_{3}\right) .
$$

For $q=27$, the cross-ratio of any 4 -set in $P G(1,27)$ is separated into five classes, $\left\{\alpha^{13}\right\},\left\{\alpha, \alpha^{2}, \alpha^{10}, \alpha^{16}, \alpha^{24}, \alpha^{25}\right\},\left\{\alpha^{3}, \alpha^{4}, \alpha^{6}, \alpha^{20}, \alpha^{22}, \alpha^{23}\right\},\left\{\alpha^{5}, \alpha^{7}, \alpha^{11}, \alpha^{15}, \alpha^{19}, \alpha^{21}\right\}, \quad$ and $\left\{\alpha^{8}, \alpha^{9}, \alpha^{12}, \alpha^{14}, \alpha^{17}, \alpha^{18}\right\}$. Let $\Gamma_{q}(n+2)$ refers to the standard frame in $P G(n, q)$. Here, $\mathcal{C}_{n, q}(f)$ is the $(n+1) \times(n+1)$ companion matrix of the projective space $P G(n, q)$ by the primitive polynomial $f$ of degree $(n+1)$. The matrix $\mathcal{C}_{n, q}(f)$ is always cyclic matrix of order $\theta(n, q)$; that is, $\mathcal{C}_{n, q}(f)$ generate a cyclic subgroup of $P G L(n+1, q)$.

If the order of $\mathcal{C}_{n, q}(f)$ is factored to $\sigma_{1}, \sigma_{2}, \ldots, \sigma_{r}$ and $\Lambda \subseteq\{1, \ldots r\}$, then the group generated by $\mathcal{C}_{n, q}(f) \prod_{j \in \Lambda} \sigma_{j}$ forms a subgroup of $\left\langle\mathcal{C}_{n, q}(f)\right\rangle$ of order $\prod_{l \in \Lambda^{c}} \sigma_{l}$. The $\prod_{l \in \Lambda^{c}} \sigma_{l}$ orbits of length $\prod_{j \in \Lambda} \sigma_{j}$ that are constructed from the of group $\left\langle\mathcal{C}_{n, q}(f)^{\prod_{j \in \Lambda} \sigma_{j}}\right\rangle$ on the $P G(n, q)$ are splitting the space $P G(n, q)$. Not all of these orbits have a known representation in the projective space. But as special orbits, for any positive integer $m$, if $m$ and $(n+1)$ coprime, then $\theta(n, q)$ divides $\theta\left(n, q^{m}\right)$ and the orbits $\operatorname{orb}(i), i=0, \ldots, \frac{\theta\left(n, q^{m}\right)}{\theta(n, q)}-1$ of $\mathcal{C}_{n, q^{m}}(f)^{\frac{\theta\left(n, q^{m}\right)}{\theta(n, q)}}$ are subgeometries $P G(n, q)$ which are equivalent, since $\operatorname{orb}(i)=\operatorname{orb}(0) \mathcal{C}_{n, q^{m}}(f)^{i}[1]$.

Throughout this paper, the symbol $\sigma_{i}^{*}$ refers to the factor of $\theta(n, q)$, such that $\theta(n, q)=\sigma_{i} \sigma_{i}^{*}$.

As mentioned before, the classification of $P G(1,27)$ was achieved by Ibrahim [7], where each $k$-set, which contains the standard frame $\Gamma_{27}(3)$, is partitioned into $k$ of projectively distinct $(k-1)$-sets, $k=5, \ldots, 14$. The results are summarized below.

Theorem 2.3: The number of inequivalent $k$-sets and their stabilizer group types in $P G(1,27)$ are summarized in Table-1.

A cell $G: m$ means that $m$ of $k$-sets is stabilized by the group of type $G ; \hat{n}_{k}$ indicates the number of inequivalent $k$-sets.

Table 1- Summary of the results of the classification of PG(1,27).

\begin{tabular}{|c|c|l|c|c|c|c|c|c|}
\hline$k$-set & $\hat{n}_{k}$ & \multicolumn{7}{|c|}{$G: m$} \\
\hline 3-set & 1 & $1: S_{3}$ & & & & & & \\
\hline 4-set & 5 & $S_{4}: 1$ & $V_{4}: 4$ & & & & & \\
\hline 5-set & 8 & \multicolumn{1}{|c|}{$I: 2$} & $Z_{2}: 6$ & & & & & \\
\hline 6-set & 34 & $I: 6$ & $Z_{2}: 14$ & $V_{4}: 6$ & $S_{3}: 4$ & & & \\
\hline 7 -set & 73 & $I: 51$ & $Z_{2}: 17$ & $S_{3}: 4$ & $D_{7}: 1$ & & & \\
\hline 8-set & 196 & $I: 128$ & $Z_{2}: 54$ & $V_{4}: 11$ & $D_{4}: 3$ & & & \\
\hline 9-set & 382 & $I: 323$ & $Z_{2}: 54$ & $Z_{3}: 4$ & & \multicolumn{2}{|c|}{$\left(Z_{3} \times Z_{3}\right) \rtimes Z_{2}: 1$} \\
\hline 10 -set & 745 & $I: 600$ & $Z_{2}: 125$ & $Z_{3}: 4$ & $V_{4}: 15$ & $\left(Z_{3} \times Z_{3}\right) \rtimes Z_{2}: 1$ \\
\hline 11 -set & 1142 & $I: 1043$ & $Z_{2}: 99$ & & & & & \\
\hline 12 -set & 1665 & $I: 1449$ & $Z_{2}: 182$ & $Z_{3}: 3$ & $V_{4}: 21$ & $S_{3}: 6$ & $D_{4}: 3$ & $\mathcal{A}_{4}: 1$ \\
\hline 13 -set & 1976 & $I: 1840$ & $Z_{2}: 125$ & $Z_{3}: 4$ & $S_{3}: 6$ & $D_{13}: 1$ & & \\
\hline 14 -set & 2170 & $I: 1924$ & $Z_{2}: 224$ & $V_{4}: 19$ & $Z_{3}: 1$ & $D_{7}: 1$ & $D_{14}: 1$ & \\
\hline
\end{tabular}

Regarding $P G(1,27)$, it has 28 points with four factors, namely $2,4,7$, and 14 . So, It can partition the projective line into two disjoint 14 -sets, seven disjoint 4-sets, and four disjoint 7 -sets. 
In this paper, all details about the splitting of the projective line of order twenty-seven into disjoint, inequivalent 4-sets and 7-sets are provided, as well as into disjoint equivalent $(E Q)$ and inequivalent $(N E Q)$ 14-sets with stabilizer groups for each splitting. Some examples related to each size of splitting are given. The idea of the splitting of line was studied in the view of orbits of action group on the line. Finally, the numbers of projectively inequivalent $k$-arcs on the conic $C^{*}=\left\{P\left(t^{2}, t, 1\right) \in P G(2,27) \mid t \in F_{27}^{+}\right\}$were founded.

All over this paper, the symbol $\delta_{i}^{n, k}, 1 \leq k \leq m$ refers to the splitter part $k$ of length $n$, index symbol $i$, and $\mathcal{S}_{i}^{n}=\left\{\mathcal{S}_{i}^{n, 1} ; \ldots, \mathcal{S}_{i}^{n, m}\right\}$, where $n$ and $m$ are factors of $\theta(1, q)$.

\section{Splitting The Projective Line by Factor 14}

A 14-set, $\mathcal{S}_{i}^{14,1}$, and its complement, $\mathcal{S}_{i}^{14,1^{c}}=\mathcal{S}_{i}^{14,2}$, formed a splitting of $P G(1,27)$. The stabilizer group $G_{\mathcal{S}_{i}^{14,1}}$ of $\mathcal{S}_{i}^{14,1}$ also fixes the complement $\delta_{i}^{14,1^{c}}$, due to the action of the projective linear group $P G L(2,27)$ on $P G(1,27)$. Thus, if $G \subseteq P G L(2,27)$ fixes a subset $A$, then it will fix $P G(1,27)-A$. If $P G(1,27)$ splitted into two 14 -sets $\mathcal{S}_{i}^{14}=\left\{\mathcal{S}_{i}^{14,1} ; \mathcal{S}_{i}^{14,2}\right\}$, then the stabilizer group of the partition $\delta_{i}^{14}$ is as follows.

(i) If $\mathcal{S}_{i}^{14,1}$ projectively inequivalent to its complement $\mathcal{S}_{i}^{14,1^{c}}$, then $G_{\delta_{i}^{14,1^{c}}}$ is $G_{\mathcal{S}_{i}^{14,1}}$ and the stabilizer group of the splitting, $\mathcal{S}_{i}^{14}$, is also $G_{\mathcal{S}_{i}^{14,1}}$. This type of splitting is called to be of type $N E Q$.

(ii) If $\mathcal{S}_{i}^{14,1}$ projectively equivalent to its complement $\mathcal{S}_{i}^{14,1^{c}}$, then the stabilizer group of the splitting, $\mathcal{S}_{i}^{14}$, is the $G_{\mathcal{S}_{i}^{14}}$ union to all the projectivity between $\mathcal{S}_{i}^{14,1}$ and $\mathcal{S}_{i}^{14,1^{c}}$. In this case, the stabilizer of the splitting, $\mathcal{S}_{i}^{14}$, is generated always by two elements, one belongs to $G_{\mathcal{S}_{i}^{14,1}}$ and the other is the projectivity between $\mathcal{S}_{i}^{14,1}$ and $\mathcal{S}_{i}^{14,1^{c}}$. This type of splitting is called to be of type $E Q$.

A mathematical Gap programming [9] was used to examine the equivalence issues between the 14sets and deduced the following theorem. For the equivalence examination procedure, see $[2,4]$.

Theorem 3.1: The projective line $P G(1,27)$ has $\delta_{i}^{14}$ splitting of type $E Q, 1 \leq i \leq 298$, and $N E Q, 1 \leq i \leq 1872$.

Table-2 provides the details of stabilizer group types of the partitions in Theorem 3.1.

Table 2- Types and numbers of the stabilizer groups to the partitions $\mathrm{S} \_\mathrm{i}^{\wedge} 14$

\begin{tabular}{|c|l|l|l|l|l|l|}
\hline$E Q$ & $260: Z_{2}$ & $27: V_{4}, 1: Z_{4}$ & $7: D_{4}$ & $1: D_{13}$ & $1: D_{14}$ & $1: G_{56}$ \\
\hline$N E Q$ & $1664: I$ & $196: Z_{2}$ & $12: V_{4}$ & & & \\
\hline
\end{tabular}

The group $G_{56}$ has 29 elements of order two, 2 elements of order four, 6 elements of order seven, 6 elements of order fourteen, and 12 elements of order twenty-eight.

\section{Example 3.2}

(i) Splitting type $E Q$

Let $\mathcal{S}_{i}^{14,1}=\left\{\infty, 0,1, \alpha^{13}, \alpha, \alpha^{2}, \alpha^{4}, \alpha^{6}, \alpha^{7}, \alpha^{8}, \alpha^{9}, \alpha^{11}, \alpha^{14}, \alpha^{15}\right\}$ be the 14 -set and $\mathcal{S}_{i}^{14,1^{c}}=$ $\left\{\alpha^{3}, \alpha^{5}, \alpha^{10}, \alpha^{12}, \alpha^{16}, \alpha^{17}, \alpha^{18}, \alpha^{19}, \alpha^{20}, \alpha^{21}, \alpha^{22}, \alpha^{23}, \alpha^{24}, \alpha^{25}\right\}$ be its complement. The stabilizer group of this partition $\delta_{i}^{14}$ is the dihedral group of order $14, D_{14}$.

$$
D_{14}=\left\langle a=\left(\frac{1}{\alpha^{11} t}\right), b=\left(\frac{t+\alpha^{16}}{\alpha^{14} t+\alpha^{23}}\right): a^{2}=b^{14}=1, b a=a b^{-1}\right\rangle .
$$

Note that $b$ is only the projective transformation from $\mathcal{S}_{i}^{14,1}$ into $\mathcal{S}_{i}^{14,1^{c}}$.

(ii) Splitting type $N E Q$

Let $\mathcal{S}_{j}^{14,1}=\left\{\infty, 0,1, \alpha^{13}, \alpha, \alpha^{2}, \alpha^{3}, \alpha^{6}, \alpha^{8}, \alpha^{9}, \alpha^{15}, \alpha^{5}, \alpha^{17}, \alpha^{23}\right\}$ be the 14-set and $\delta_{j}^{14,1^{c}}=$ $\left\{\alpha^{4}, \alpha^{7}, \alpha^{10}, \alpha^{11}, \alpha^{12}, \alpha^{14}, \alpha^{16}, \alpha^{18}, \alpha^{19}, \alpha^{20}, \alpha^{21}, \alpha^{22}, \alpha^{24}, \alpha^{25}\right\}$ be its complement. Here, $\mathcal{S}_{i}^{14,1}$ and $\delta_{j}^{14,1^{c}}$ are projectively inequivalent and the group that fixes the partition $\delta_{j}^{14}$ is the Klein fourgroup, $\mathrm{V}_{4}=\left\langle c=\left(\frac{\alpha^{13} t+1}{\alpha^{10} t+1}\right), d=\left(\frac{\alpha^{16} t+1}{\alpha^{8} t+\alpha^{3}}\right): c^{2}=d^{2}=1\right\rangle$. 


\section{Splitting the Projective Line by Factor 7}

The $P G(1,27)$ can be split into four 7-sets, $\mathcal{S}_{i}^{7}=\left\{\mathcal{S}_{i}^{7,1}, \mathcal{S}_{i}^{7,2}, \mathcal{S}_{i}^{7,3}, \mathcal{S}_{i}^{7,4}\right\}$. There are many possibilities to achieve that, one of which is as given below:

$$
\begin{array}{lll}
\mathcal{S}_{i}^{7,1}=\left\{\infty, 0,1, \alpha, \alpha^{2}, \alpha^{3}, \alpha^{4}\right\}, & \mathcal{S}_{i}^{7,2}= & \left\{\alpha^{5}, \alpha^{6}, \alpha^{7}, \alpha^{8}, \alpha^{9}, \alpha^{10}, \alpha^{11}\right\}, \\
\mathcal{S}_{i}^{7,3}=\left\{\alpha^{12}, \alpha^{13}, \alpha^{14}, \alpha^{15}, \alpha^{16}, \alpha^{17}, \alpha^{18}\right\}, & \mathcal{S}_{i}^{7,4}= & \left\{\alpha^{19}, \alpha^{20}, \alpha^{21}, \alpha^{22}, \alpha^{23}, \alpha^{24}, \alpha^{25}\right\} .
\end{array}
$$

Each of these four 7-sets is equivalent by a matrix transformation $T$ to one that passes through the

\begin{tabular}{|c|c|c|c|c|c|}
\hline & & $T=$ Row 1, Row 2 & & & $T=$ Row 1, Row 2 \\
\hline $\mathcal{H}_{1}$ & $\mathcal{S}_{1}^{7}$ & $1 \quad 0,0 \quad 1$ & $\mathcal{H}_{2}$ & $\mathcal{S}_{2}^{7}$ & $\alpha^{8} \quad \alpha^{3}, \alpha^{14} \quad \alpha^{4}$ \\
\hline $\mathcal{H}_{2}$ & $\mathcal{S}_{3}^{7}$ & $\alpha^{22} \quad \alpha^{10}, \alpha^{2} \quad \alpha^{11}$ & $\mathcal{H}_{2}$ & $\mathcal{S}_{4}^{7}$ & $\alpha^{10} \quad \alpha^{17}, \alpha^{16} \quad \alpha^{18}$ \\
\hline
\end{tabular}
standard frame $\Gamma_{27}(3)$, as shown below.

where $\mathcal{H}_{1}=\Gamma_{27}(3) \cup\left\{\alpha, \alpha^{2}, \alpha^{3}, \alpha^{4}\right\}$ and $\mathcal{H}_{2}=\Gamma_{27}(3) \cup\left\{\alpha, \alpha^{6}, \alpha^{7}, \alpha^{12}\right\}$. Both of the 7-sets, $\mathcal{H}_{1}$ and $\mathcal{H}_{2}$, have three distinct pairs of 6-sets and stabilizer groups of type $Z_{2}$.

\section{Splitting the Projective Line by Factor 4}

There are five distinct 4-sets, called the 4-sets of types $H, N_{1}, N_{2}, N_{3}$, and $N_{4}$, where

$$
\begin{array}{lll}
H= & \Gamma_{27}(3) \bigcup\left\{\alpha^{13}\right\}, & N_{1}=\Gamma_{27}(3) \bigcup\{\alpha\}, \\
N_{3}=\Gamma_{27}(3) \bigcup\left\{\alpha^{5}\right\}, & N_{4}=\Gamma_{27}(3) \bigcup\left\{\alpha^{8}\right\} .
\end{array}
$$

These types emerge according to the cross-ratio of each 4 -set. From these 4 -sets, the projective line can be divided into seven 4-sets, as displayed below. These 4-sets are found with comprehensive details in [7].

(i) Partitioning by $\mathcal{S}_{1}^{4}, \mathcal{S}_{1}^{4, i} \cong_{p} H, i=1,2, \ldots 7$.

$$
\begin{array}{ccc}
\mathcal{S}_{1}^{4,1}=\Gamma_{27}(3) \cup\left\{\alpha^{13}\right\}, & \mathcal{S}_{1}^{4,2}=\left\{\alpha, \alpha^{2}, \alpha^{3}, \alpha^{15}\right\}, & \mathcal{S}_{1,3}^{4,3}=\left\{\alpha^{4}, \alpha^{5}, \alpha^{6}, \alpha^{18}\right\}, \\
\mathcal{S}_{1}^{4,4}=\left\{\alpha^{7}, \alpha^{8}, \alpha^{16}, \alpha^{19}\right\}, & \mathcal{S}_{1,5}^{4,5}=\left\{\alpha^{9}, \alpha^{12}, \alpha^{20}, \alpha^{21}\right\}, & \mathcal{S}_{1}^{4,6}=\left\{\alpha^{10}, \alpha^{14}, \alpha^{17}, \alpha^{22}\right\}, \\
& \mathcal{S}_{1}^{4,7}=\left\{\alpha^{11}, \alpha^{23}, \alpha^{24}, \alpha^{25}\right\} . &
\end{array}
$$

(ii) Partitioning by $\mathcal{S}_{2}^{4}, \mathcal{S}_{2}^{4, i} \cong_{p} N_{1}, i=1,2, \ldots 7$.

$$
\begin{array}{ccc}
\mathcal{S}_{2}^{4,1}=\Gamma_{27}(3) \cup\{\alpha\}, & \mathcal{S}_{2}^{4,2}=\left\{\alpha^{2}, \alpha^{3}, \alpha^{4}, \alpha^{9}\right\}, & \mathcal{S}_{2}^{4,3}=\left\{\alpha^{5}, \alpha^{6}, \alpha^{7}, \alpha^{12}\right\}, \\
\mathcal{S}_{2}^{4,4}=\left\{\alpha^{8}, \alpha^{10}, \alpha^{11}, \alpha^{19}\right\}, & \mathcal{S}_{2}^{4,5}=\left\{\alpha^{13}, \alpha^{14}, \alpha^{15}, \alpha^{20}\right\}, & \mathcal{S}_{2}^{4,6}=\left\{\alpha^{16}, \alpha^{17}, \alpha^{18}, \alpha^{23}\right\}, \\
\mathcal{S}_{2}^{4,7}=\left\{\alpha^{21}, \alpha^{22}, \alpha^{24}, \alpha^{25}\right\} . &
\end{array}
$$

(iii) Partitioning by $\mathcal{S}_{3}^{4}, \mathcal{S}_{3}^{4, i} \cong_{p} N_{2}, i=1,2, \ldots 7$.

$$
\begin{array}{ccc}
\mathcal{S}_{3}^{4,1}=\Gamma_{27}(3) \cup\left\{\alpha^{3}\right\}, & \mathcal{S}_{3}^{4,2}=\left\{\alpha, \alpha^{2}, \alpha^{4}, \alpha^{18}\right\}, & \mathcal{S}_{3}^{4,3}=\left\{\alpha^{5}, \alpha^{6}, \alpha^{7}, \alpha^{10}\right\}, \\
\mathcal{S}_{3}^{4,4}=\left\{\alpha^{8}, \alpha^{9}, \alpha^{11}, \alpha^{25}\right\}, & \mathcal{S}_{3}^{4,5}=\left\{\alpha^{12}, \alpha^{13}, \alpha^{14}, \alpha^{17}\right\}, & \mathcal{S}_{3}^{4,6}=\left\{\alpha^{15}, \alpha^{16}, \alpha^{20}, \alpha^{21}\right\}, \\
\mathcal{S}_{3}^{4,7}=\left\{\alpha^{19}, \alpha^{22}, \alpha^{23}, \alpha^{24}\right\} . &
\end{array}
$$

(iv) Partitioning by $\mathcal{S}_{4}^{4}, \mathcal{S}_{4}^{4, i} \cong_{p} N_{3}, i=1,2, \ldots 7$.

$$
\begin{array}{cl}
\mathcal{S}_{4}^{4,1}=\Gamma_{27}(3) \cup\left\{\alpha^{5}\right\}, & \mathcal{S}_{4}^{4,2}=\left\{\alpha, \alpha^{2}, \alpha^{3}, \alpha^{7}\right\}, \\
\mathcal{S}_{4}^{4,4}=\left\{\alpha^{9}, \alpha^{10}, \alpha^{11}, \alpha^{13}\right\}, & \mathcal{S}_{4}^{4,5}=\left\{\alpha^{12}, \alpha^{14}, \alpha^{15}, \alpha^{17}\right\}, \\
\mathcal{S}_{4}^{4,7}=\left\{\alpha^{21}, \alpha^{23}, \alpha^{24}, \alpha^{25}\right\} . &
\end{array}
$$$$
\mathcal{S}_{4}^{4,3}=\left\{\alpha^{4}, \alpha^{6}, \alpha^{8}, \alpha^{16}\right\}
$$$$
\mathcal{S}_{4}^{4,6}=\left\{\alpha^{18}, \alpha^{19}, \alpha^{20}, \alpha^{21}\right\} \text {, }
$$

(v) Partitioning by $\delta_{5}^{4}, \delta_{5}^{4, i} \cong_{p} N_{3}, i=1,2, \ldots 7$.

$$
\begin{aligned}
\mathcal{S}_{5}^{4,1}=\Gamma_{27}(3) \cup\left\{\alpha^{8}\right\}, & \mathcal{S}_{5}^{4,2}=\left\{\alpha, \alpha^{2}, \alpha^{3}, \alpha^{4}\right\}, & \mathcal{S}_{5}^{4,3}=\left\{\alpha^{5}, \alpha^{6}, \alpha^{7}, \alpha^{16}\right\}, \\
\mathcal{S}_{5}^{4,4}=\left\{\alpha^{9}, \alpha^{10}, \alpha^{11}, \alpha^{12}\right\}, & \mathcal{S}_{5}^{4,5}=\left\{\alpha^{13}, \alpha^{14}, \alpha^{15}, \alpha^{24}\right\}, & \mathcal{S}_{5}^{4,6}=\left\{\alpha^{17}, \alpha^{18}, \alpha^{23}, \alpha^{25}\right\}, \\
\mathcal{S}_{5}^{4,7}=\left\{\alpha^{19}, \alpha^{20}, \alpha^{21}, \alpha^{22}\right\} . & &
\end{aligned}
$$

It is also possible to divide $P G(1,27)$ into seven 4 -sets that contain all the five types of 4 -sets, as shown below.

(vi) Partitioninng by $\mathcal{S}_{6}^{4}, \mathcal{S}_{6}^{4, i} \cong_{p} H, i=1,6,7$ and $\mathcal{S}_{6}^{4, j} \cong_{p} N_{j}, j=2,3,4,5$.

$$
\begin{array}{rll}
\mathcal{S}_{6}^{4,1}=\Gamma_{27}(3) \cup\left\{\alpha^{13}\right\}, & \mathcal{S}_{6}^{4,2}=\left\{\alpha, \alpha^{2}, \alpha^{3}, \alpha^{8}\right\}, & \mathcal{S}_{6}^{4,3}=\left\{\alpha^{4}, \alpha^{5}, \alpha^{6}, \alpha^{9}\right\}, \\
\mathcal{S}_{6}^{4,4}=\left\{\alpha^{7}, \alpha^{10}, \alpha^{11}, \alpha^{14}\right\}, & \mathcal{S}_{6}^{4,5}=\left\{\alpha^{15}, \alpha^{19}, \alpha^{22}, \alpha^{25}\right\}, & \mathcal{S}_{6}^{4,6}=\left\{\alpha^{12}, \alpha^{17}, \alpha^{20}, \alpha^{24}\right\}, \\
\mathcal{S}_{6}^{4,7}=\left\{\alpha^{16}, \alpha^{18}, \alpha^{21}, \alpha^{23}\right\} . & &
\end{array}
$$




\section{Splitting by Orbits}

By taking the companion matrix $T$ of the projective line $P G(1,27)$, we have

$T=\mathcal{C}_{1,27}(f)=\left[\begin{array}{ccc}1 & 0 & 0 \\ 0 & 1 & 0 \\ \alpha^{15} & \alpha^{14} & 0\end{array}\right]$.

The order of $T$ is 28 , so the factors of the order are $\sigma_{1}=2, \sigma_{2}=4, \sigma_{3}=7$, and $\sigma_{4}=14$. Let $\operatorname{orb}_{\sigma_{i}}(j)$ denotes the orbit of $T^{\sigma_{i}}, j=0, \ldots \sigma_{i}-1$.

Theorem 6.1: The projective line $P G(1,27)$ can be split by orbits into:

(i) seven 4-sets of type $H$ which form $P G(1,3)$;

(ii) two 14-sets of type $E Q$;

(iii) four 7-sets that are projectively equivalent to a unique 7-set through the standard frame;

(iv) fourteen pairs such that any two distinct pairs form a 4-set of type $N_{3}$.

Proof:

(i) Case $\sigma_{3}=7$ : As explained in Section 2, the orbits $\operatorname{orb}_{7}(j), j=0, \ldots, 7$ of $T^{7}$ are just the $P G(1,3)$ and all of them are 4-sets of type $H$ in $P G(1,27)$; that is, $C R\left(\operatorname{orb}_{7}(j)\right)=\alpha^{14}$.

$$
\begin{array}{cccc}
\operatorname{orb}_{7}(0)= & \left\{\infty, \alpha^{23}, \alpha^{22}, \alpha^{18}\right\}, & \operatorname{orb}_{7}(4)= & \left\{\alpha^{3}, \alpha^{24}, \alpha^{5}, 1\right\}, \\
\operatorname{orb}_{7}(1)= & \left\{0, \alpha^{11}, \alpha^{7}, \alpha^{6}\right\}, & \operatorname{orb}_{7}(5)= & \left\{\alpha^{16}, \alpha^{25}, \alpha^{17}, \alpha^{2}\right\}, \\
\operatorname{orb}_{7}(2)= & \left\{\alpha^{20}, \alpha^{21}, \alpha^{10}, \alpha^{14}\right\}, & \operatorname{orb}_{7}(6)= & \left\{\alpha^{15}, \alpha^{19}, \alpha^{8}, \alpha^{9}\right\} . \\
\operatorname{orb}_{7}(3)= & \left\{\alpha, \alpha^{12}, \alpha^{4}, \alpha^{13}\right\}, & &
\end{array}
$$

(ii) Case $\sigma_{1}=2$ : The two orbits, $\operatorname{orb}_{2}(0)$ and $\operatorname{orb}_{2}(1)$, of $T^{2}$ are 14-sets that do not have a standard frame and both are projectively equivalent to the unique 14-set of type $E Q, \mathcal{S}_{i}^{14,1}$, with stabilizer group $G_{56}$ in Theorem 3.1; that is, these orbits are of type $E Q$.

$$
\begin{array}{cc}
\mathcal{S}_{i}^{14,1}= & \Gamma_{27}(3) \cup\left\{\alpha^{13}, \alpha, \alpha^{2}, \alpha^{3}, \alpha^{6}, \alpha^{14}, \alpha^{15}, \alpha^{10}, \alpha^{16}, \alpha^{19}, \alpha^{23}\right\}, \\
\operatorname{orb}_{2}(0)= & \left\{\infty, \alpha^{20}, \alpha^{3}, \alpha^{15}, \alpha^{11}, \alpha^{12}, \alpha^{25}, \alpha^{22}, \alpha^{10}, \alpha^{5}, \alpha^{8}, \alpha^{6}, \alpha^{13}, \alpha^{2}\right\}, \\
\operatorname{orb}_{2}(1)= & \left\{0, \alpha, \alpha^{16}, \alpha^{23}, \alpha^{21}, \alpha^{24}, \alpha^{19}, \alpha^{7}, \alpha^{4}, \alpha^{4}, \alpha^{17}, \alpha^{18}, \alpha^{14}, 1, \alpha^{9}\right\} .
\end{array}
$$

The matrix transformation from $T_{i}: \delta_{i}^{14,1} \rightarrow \operatorname{orb}_{2}(i), i=0,1$ is represented by its rows, as follows:

$$
T_{0}=\left[\alpha^{4} 0, \alpha^{22} 1\right], \quad T_{1}=\left[0 \alpha^{3}, \alpha^{15} \alpha^{8}\right] .
$$

(iii) Case $\sigma_{2}=4$ : The four orbits, $\operatorname{orb}_{4}(0), \ldots, \operatorname{orb}_{4}(6)$, of $T^{4}$ are 7-sets that do not have a standard frame and all are projectively equivalent to the unique 7 -set, $\mathcal{H}_{3}$, and projectively inequivalent from $\mathcal{H}_{1}$ and $\mathcal{H}_{2}$, with a stabilizer group, of type dihedral group, of degree seven that is given in Table-1.

$$
\begin{array}{cccc}
\mathcal{H}_{3}= & \multicolumn{1}{c}{\Gamma_{27}(3) \cup\left\{\alpha, \alpha^{6}, \alpha^{11}, \alpha^{12}\right\},} \\
\operatorname{orb}_{4}(0)= & \left\{\infty, \alpha^{3}, \alpha^{11}, \alpha^{25}, \alpha^{10}, \alpha^{8}, \alpha^{13}\right\}, & \operatorname{orb}_{4}(2)= & \left\{\alpha^{20}, \alpha^{15}, \alpha^{12}, \alpha^{22}, \alpha^{5}, \alpha^{6}, \alpha^{2}\right\}, \\
\operatorname{orb}_{4}(1)= & \left\{0, \alpha^{16}, \alpha^{21}, \alpha^{19}, \alpha^{4}, \alpha^{18}, 1\right\}, & \operatorname{orb}_{4}(3)= & \left\{\alpha, \alpha^{23}, \alpha^{24}, \alpha^{7}, \alpha^{17}, \alpha^{14}, \alpha^{9}\right\} .
\end{array}
$$

The matrix transformation from $L_{i}: \mathcal{H}_{3} \rightarrow \operatorname{orb}_{4}(i), i=0, \ldots, 3$ is represented by its rows, as follows: $L_{0}=\left[\begin{array}{ll}\alpha^{20} 0, \alpha^{25} 1\end{array}\right], \quad L_{2}=\left[\begin{array}{lll}\alpha^{4} & \alpha^{10}, \alpha^{7} & \alpha^{11}\end{array}\right], \quad L_{1}=\left[\begin{array}{llll}0 & \alpha^{5}, \alpha & \alpha^{8}\end{array}\right], \quad L_{2}=\left[\begin{array}{lll}\alpha^{13} & \alpha^{12}, \alpha^{14} & \alpha^{7}\end{array}\right]$.

(iv) Case $\sigma_{4}=14$ : The fourteen orbits, $\operatorname{orb}_{14}(0), \ldots$, orb $b_{14}(13)$ of $T^{14}$ are 2 -sets; that is, $P G(1,27)$ is separated into fourteen order pairs.

$$
\begin{array}{ccccccc}
\left(\infty \alpha^{22}\right) & \left(0 \alpha^{7}\right) & \left(\alpha^{20} \alpha^{10}\right) & \left(\alpha \alpha^{4}\right) & \left(\alpha^{3} \alpha^{5}\right) & \left(\alpha^{16} \alpha^{17}\right) & \left(\alpha^{15} \alpha^{8}\right) \\
\left(\alpha^{23} \alpha^{18}\right) & \left(\alpha^{11} \alpha^{6}\right) & \left(\alpha^{21} \alpha^{14}\right) & \left(\alpha^{12} \alpha^{13}\right) & \left(\alpha^{24} 1\right) & \left(\alpha^{25} \alpha^{2}\right) & \left(\alpha^{19} \alpha^{9}\right)
\end{array}
$$

Let us code the five types of 4-sets by 0 for $H, 1$ for $N_{1}, 2$ for $N_{2}, 3$ for $N_{3}$, and 4 for $N_{4}$. Each $i$-th row of the matrix $\mathcal{M}$ comes from the type of 4 -set that is constructed from the $i$-th pair with the other 13 pairs in orders. The interesting properties of $\mathcal{M}$ are:

(1) The $(i+1)$-th row is just the cyclic of the $i$-th row.

(2) If any row of the matrix $\mathcal{M}$ is deleted, then the new matrix is a square matrix with diagonal 3. Therefore, the line $P G(1,27)$ is separated into fourteen pairs such that any two of them construct a 4set of type $N_{3}$. 


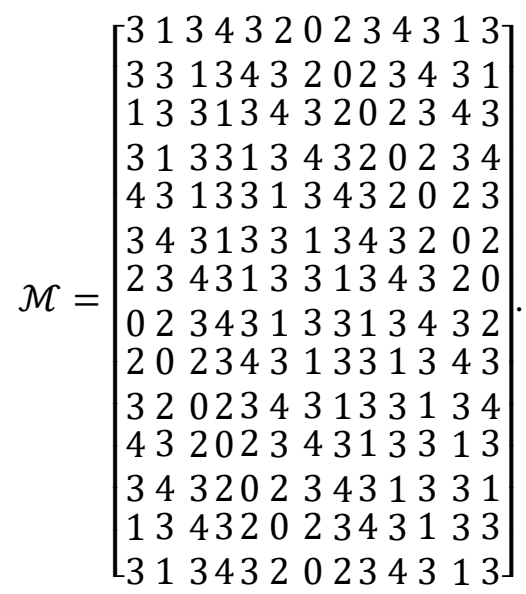

\section{Arcs on the conic}

In the projective plane over $F_{q}$, the zero set of a non-singular form $F$ of degree two is called a conic and denoted by $C=v(F)$. If a conic passes through the standard frame $\Gamma_{q}(4)$, then $F=$ $a_{1} X_{0} X_{1}+a_{2} X_{0} X_{2}+a_{3} X_{1} X_{2}$. Each projectively inequivalent 5 -arc determines a unique conic and the conic is unique up to projectivity. The form $F^{*}=X_{1}^{2}-X_{0} X_{2}$ has the four points of $\Gamma_{q}(4)$ as zero points, and the conic $C^{*}=v\left(F^{*}\right)$ is parameterized such that $C^{*}=\left\{P\left(t^{2}, t, 1\right) \in P G(2, q) \mid t \in F_{q}^{+}\right\}$ corresponding to $P G(1, q)$ by

$$
\varphi^{*}: P G(1, q) \rightarrow C^{*} ; P(X, Y) \mapsto P\left((X / Y)^{2}, X / Y, 1\right) .
$$

If the map $\varphi^{*}$ is restricted on the set of all inequivalent $k$-sets that contains $\Gamma_{q}(3), \Gamma_{1}(k, q)$, then the image will be the set of all incomplete, inequivalent $k$-arcs that contains $\Gamma_{q}(4)$ on the conic $C^{*}$ up to projectivity. Let us denote $\varphi^{*}\left(\Gamma_{1}(k, q)\right)$ by $C_{2}^{*}(k, q)$. Then, we can define a bijective map $\varphi_{k}^{*}=\left.\varphi^{*}\right|_{\Gamma_{1}(k, q)}: \Gamma_{1}(k, q) \rightarrow C_{2}^{*}(k, q)$.

Throughout this paper, the companion matrix $\mathcal{C}_{2,27}(f)=\left[\begin{array}{ccc}1 & 0 & 0 \\ 0 & 1 & 0 \\ \alpha^{15} & \alpha^{14} & 0\end{array}\right]$ is used to construct the points and lines of $P G(2,27)$, where $\Gamma_{27}(4)=\{1,2,3,487\}$ is the standard frame in numeral form. Also, the position representations of points of $P G(2,27)$ will be used.

The following results are deduced from the above and Theorem 2.3 .

Theorem 7.1: In $P G(1,27)$, there are incomplete $k$-arcs through the standard frame, $k=5, \ldots, 14$, on the conic $C^{*}$.

\section{Proof:}

Firstly, the map $\varphi_{4}^{*}=\left.\varphi^{*}\right|_{\Gamma_{1}(k, 27)}: \Gamma_{1}(k, q) \rightarrow C_{2}^{*}(k, 27)$ transforms the five 4-sets into a unique 4arc, which is the frame $\Gamma_{27}(4)$, since all the 4-arcs are projectively equivalent. Secondly, using the $\operatorname{map} \varphi_{k}^{*}=\left.\varphi^{*}\right|_{\Gamma_{1}(k, 27)}: \Gamma_{1}(k, q) \rightarrow C_{2}^{*}(k, 27)$, eight 5 -arcs are constructed from eight 5 -sets, $\mathcal{P}_{i}$, in $P G(1,27)$, as founded in [7], where

$$
\begin{array}{rc}
\varphi_{5}^{*}\left(\mathcal{P}_{1}\right)=\varphi_{5}^{*}(E \cup\{\alpha\})=A_{1}=\Gamma_{27}(4) \cup\{6\}, & \varphi_{5}^{*}\left(\mathcal{P}_{5}\right)=\varphi_{5}^{*}\left(N_{1} \cup\left\{\alpha^{7}\right\}\right)=A_{5}=\Gamma_{27}(4) \bigcup\{13\}, \\
\varphi_{5}^{*}\left(\mathcal{P}_{2}\right)=\varphi_{5}^{*}\left(N_{1} \cup\left\{\alpha^{2}\right\}\right)=A_{2}=\Gamma_{27}(4) \bigcup\{7\}, & \varphi_{5}^{*}\left(\mathcal{P}_{6}\right)=\varphi_{5}^{*}\left(N_{1} \cup\left\{\alpha^{12}\right\}\right)=A_{6}=\Gamma_{27}(4) \bigcup\{14\}, \\
\varphi_{5}^{*}\left(\mathcal{P}_{3}\right)=\varphi_{5}^{*}\left(N_{1} \bigcup\left\{\alpha^{3}\right\}\right)=A_{3}=\Gamma_{27}(4) \bigcup\{8\}, & \varphi_{5}^{*}\left(\mathcal{P}_{7}\right)=\varphi_{5}^{*}\left(N_{2} \bigcup\left\{\alpha^{7}\right\}\right)=A_{7}=\Gamma_{27}(4) \bigcup\{25\}, \\
\varphi_{5}^{*}\left(\mathcal{P}_{4}\right)=\varphi_{5}^{*}\left(N_{1} \bigcup\left\{\alpha^{6}\right\}\right)=A_{4}=\Gamma_{27}(4) \bigcup\{12\}, & \varphi_{5}^{*}\left(\mathcal{P}_{8}\right)=\varphi_{5}^{*}\left(N_{2} \bigcup\left\{\alpha^{9}\right\}\right)=A_{8}=\Gamma_{27}(4) \bigcup\{32\}
\end{array}
$$

Each 5-src, $A_{i}$, determines a form $F_{i}$ of a unique conic $C_{i}$, as given below with parameterizations.

\begin{tabular}{l|l}
\multicolumn{1}{c|}{$F_{i}$ form } & \multicolumn{1}{c}{$P(t)$ point of $C_{i}, t \in F_{27}^{+}$} \\
\hline$F_{1}=X_{0} X_{1}+\alpha^{6} X_{0} X_{2}+\alpha^{24} X_{1} X_{2}$ & $P\left(\left(t^{2}-\alpha^{14} t\right), \alpha^{10}\left(1-\alpha^{12} t\right), \alpha^{16} t\right)$ \\
$F_{2}=X_{0} X_{1}+\alpha^{3} X_{0} X_{2}+\alpha^{14} X_{1} X_{2}$ & $P\left(\alpha^{16}\left(t^{2}-\alpha^{14} t\right), \alpha^{7}\left(1-\alpha^{12} t\right), \alpha^{16} t\right)$ \\
$F_{3}=X_{0} X_{1}+\alpha^{10} X_{0} X_{2}+\alpha^{19} X_{1} X_{2}$ & $P\left(\alpha^{21}\left(t^{2}-\alpha^{14} t\right), \alpha^{14}\left(1-\alpha^{12} t\right), \alpha^{16} t\right)$ \\
$F_{4}=X_{0} X_{1}+\alpha^{7} X_{0} X_{2}+\alpha^{17} X_{1} X_{2}$ & $P\left(\alpha^{19}\left(t^{2}-\alpha^{14} t\right), \alpha^{11}\left(1-\alpha^{12} t\right), \alpha^{16} t\right)$ \\
$F_{5}=X_{0} X_{1}+\alpha^{15} X_{0} X_{2}+\alpha^{12} X_{1} X_{2}$ & $P\left(\alpha^{14}\left(t^{2}-\alpha^{14} t\right), \alpha^{19}\left(1-\alpha^{12} t\right), \alpha^{16} t\right)$ \\
$F_{6}=X_{0} X_{1}+\alpha^{16} X_{0} X_{2}+\alpha^{9} X_{1} X_{2}$ & $P\left(\alpha^{11}\left(t^{2}-\alpha^{14} t\right), \alpha^{20}\left(1-\alpha^{12} t\right), \alpha^{16} t\right)$ \\
$F_{7}=X_{0} X_{1}+\alpha^{10} X_{0} X_{2}+\alpha^{19} X_{1} X_{2}$ & $P\left(\alpha^{3}\left(t^{2}-\alpha^{14} t\right),\left(1-\alpha^{12} t\right), \alpha^{16} t\right)$ \\
$F_{8}=F_{1}=X_{0} X_{1}+\alpha^{6} X_{0} X_{2}+\alpha^{24} X_{1} X_{2}$ & $P\left(\left(t^{2}-\alpha^{14} t\right), \alpha^{10}\left(1-\alpha^{12} t\right), \alpha^{16} t\right)$ \\
\hline
\end{tabular}


Since all $C_{i}$ are projectively equivalent to $C^{*}$, then the number of incomplete, projectively inequivalent $k$-arcs, $k=5, \ldots, 14$, and their stabilizer group types are as in Theorem 2.3.

\section{Conclusions}

The projective line of order twenty seven was partitioned by disjoint $n$-sets, $n=2,4,7,14$, not all of which being of the same type, using the classification of line, where these partitions are not unique. By the factors of line order, 28, which are $2,4,7,14$, and using the action of group properties, the line was also partitioned as equivalent classes. Each of these classes is projectively equivalent to a unique type of $k$-set, $k=4,7,14$. At factor two, the line was partitioned into 14 pairs such that any two of them formed a 4-set of type $N_{3}$. The results provided in this paper can be used in the areas of graph theory, cryptography, and coding theory, see $[2,4,10,11]$.

\section{References}

1. Hirschfeld, J.W.P. 1998. Projective geometries over finite fields. Second Edition. New York: Oxford Mathematical Monographs, The Clarendon Press, Oxford University Press.

2. Al-Zangana, E.B. and Hirschfeld, J.W.P. 2016. Classification of the projective line of order nineteen and its application to error-correcting codes. 2013. 2nd International conference of mathematics and its applications, October 23-24, Al-Basra University, Iraq, 34:196-211.

3. Al-Seraji, N.A. 2014. Classification of the projective line over Galois field of order sixteen. AlMustansiriyah J. Sci., 25(1):119-128.

4. Al-Seraji, N.A. 2010. The geometry of the plane of order seventeen and its application to errorcorrecting codes. Ph.D. Thesis, University of Sussex, Brighton, UK.

5. Al-Zangana, E.B. 2016. Results in projective geometry $P G(r, 23), r=1,2$. Iraqi Journal of Science, 57(2A):964-971.

6. Al-Zangana, E.B. and Shehab, E.A. 2018. Classification of $k$-sets in $P G(1,25)$, for $k=4, \ldots, 13$. Iraqi Journal of Science, 59(1B):360-368.

7. Ibrahim, M.M. and Al-Zangana, E.B. 2021. Classification of subsets in finite projective line over Galois field of order twenty-seven. J. Phys.: Conf. Ser. 1818(1) 012087.

8. Coolsaet, K. and Sticker, H. 2011. The complete $k$-arcs of $P G(2,27)$ and $P G(2,29)$. Journal of Combinatorial Designs, 19(2): 111-130.

9. The GAP Group. 2020. GAP. Reference manual. [Online]. https://www.gap-system.org/.

10. Hirschfeld, J.W.P. and Thas, J.A. 2015. Open problems in finite projective spaces. Finite Fields and Their Applications, 32:44-81.

11. Klein, A. and Storme, L. 2011. Applications of finite geometry in coding theory and cryptography. In NATO Science for Peace and Security, Series D: Information and Communication Security Croatia, 29:38-58. 